INT. J. REMOTE SENSING, 2001, VOL. 22, NO. 17, 3619-3624

\title{
A method for the correction of AVHRR onboard IR calibration in the event of short-term radiative contamination
}

\author{
A. P. TRISHCHENKO $\dagger$ and Z. LI† \\ $\dagger$ Canada Centre for Remote Sensing, Natural Resources Canada, Ottawa, \\ Ontario, Canada, K1A 0Y7 \\ $\$$ University of Maryland, ESSIC, College Park, MD, 20742, USA
}

(Received 4 January 2001; in final form 26 April 2001)

\begin{abstract}
We analysed the operational infrared (IR) calibration of the AVHRR radiometers onboard NOAA-12, 14 and 15 satellites. It was shown that solar blackbody contamination affects the calibration of all IR channels. This source of error in the calibration may result in uncertainties in the estimation of sea surface temperature (SST) up to $0.5 \mathrm{~K}$ or more. We propose an approach to correct this error using a Fourier transform filtering technique.
\end{abstract}

\section{Introduction}

The Advanced Very High Resolution Radiometer (AVHRR) aboard the National Oceanic \& Atmospheric Administration's (NOAA) satellites is perhaps the most widely used sensor for monitoring global processes from space (Cracknell 1997). One of the few products estimated operationally from AVHRR is the SST. For climate change studies and other applications, accuracy requirement for SST is $\sim \pm(0.1 \mathrm{deg} \mathrm{K}-0.2 \mathrm{deg} \mathrm{K})$ (Allen et al. 1994, Kidwell 1998). This demands a high accuracy in the calibration of AVHRR IR measurements. This study is concerned with the calibration of AVHRR IR channels $3(3.55-3.93 \mu \mathrm{m}), 4(10.3-11.3 \mu \mathrm{m})$ and $5(11.5-12.5 \mu \mathrm{m})$.

AVHRR thermal calibration is achieved in-flight by observing the signal $C_{I C T}$ from onboard internal calibration target (ICT) and deep-space (SP) signal $C_{S P}$. Radiation from the ICT $\left(\mathbf{R}_{I C T}^{(i)}\right)$ at a temperature $\mathbf{T}_{I C T}$ can be computed by integrating Planck's function over spectral band $i$. Four platinum resistance thermometers (PRTs) are employed to measure $\mathbf{T}_{I C T}$. Gain $\mathbf{G}^{(i)}$ and offset $\mathbf{I}^{(i)}$ are defined as

$$
\begin{gathered}
G^{(i)}=\frac{\mathbf{R}_{I C T}^{(i)}-\mathbf{R}_{S P}^{(i)}}{\mathbf{C}_{T C T}^{(i)}-\mathbf{C}_{S P}^{(i)},} \\
\mathbf{I}^{(i)}=R_{S P}^{(i)}-G^{(i)}-C_{S P}^{(i)}
\end{gathered}
$$

A small non-zero radiance $\mathbf{R}_{S P}^{(i)}$ is assigned to the SP signal to account for the nonlinearity effect (Kidwell 1998).

Certain knowledge is available about the accuracy of the pre-flight IR calibration (Brown et al. 1985, 1993, Kidwell 1998, Walton et al. 1998), but much less is known 
about the accuracy of the on-board calibration. During in-flight operation, the radiometer experiences periodic temperature oscillations. Another type of variation called solar blackbody contamination (Kidwell 1998) occurs when Sun light impinges periodically the interior of the AVHRR as described by Steyn-Ross et al. (1992) for AVHRR/NOAA-11. So far, this effect has been accounted for in channel 3 calibration only. However, as shown here, the solar contamination also affects the calibration of channels 4 and 5. Our study employs global area coverage (GAC) AVHRR data obtained from the NOAA satellite active archive for NOAA-12, 14 and 15.

\section{In-flight calibration}

Pre-launch calibration was conducted with radiometer in a state of thermal equilibrium. For example, the temperature of the NOAA-14 AVHRR radiometer was changed by heating or cooling for 4 hours followed by 8 hours of thermal relaxation to reach thermal equilibrium before calibration begins. It took about 30 minutes to change the temperature of the external blackbody (Walton et al. 1998).
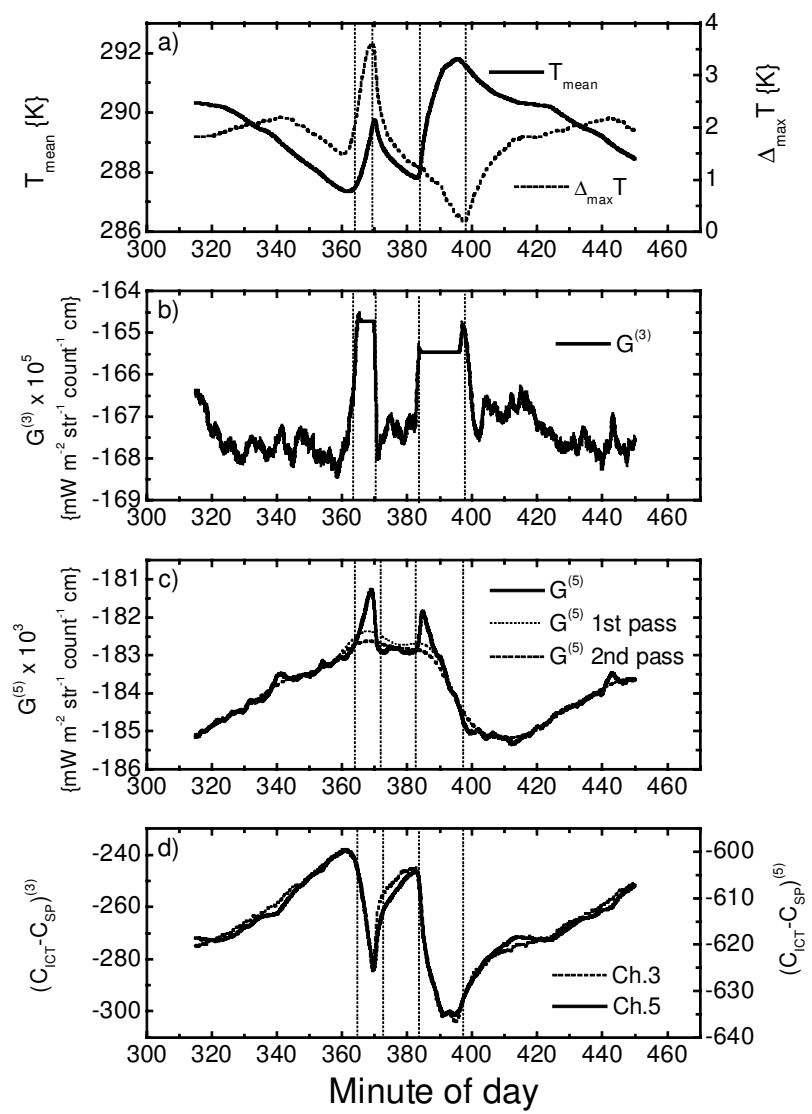

Figure 1. Mean ICT temperature $T_{\text {mean }}$ (solid) and its range $\Delta_{\max } T$ (dashed) between four PRT measurements $(a)$, gains for channel $3(b)$, and $5(c)$ and the $\left(C_{I C T}-C_{S P}\right)$ difference $(d)$ for channel 3 and 5 of AVHRR/NOAA-12 on 1 August 1995. The corrected gains $\mathbf{G}^{(5)}$ are also shown on panel $(c)$ as dashed and dotted lines. Periods of solar blackbody contamination are marked by the vertical dotted lines. 
In thermal equilibrium, the ICT temperature is expected to be equal to the radiometer and the PRT temperature.

It is a different situation for in-flight calibration, which takes place at variable temperatures as shown in figures 1-3 for AVHRR/NOAA-12, 14 and 15, respectively. The top panels $(a)$ present the mean ICT temperatures $\left(T_{\text {mean }}\right)$ computed by averaging four PRT-based temperatures (solid curves), and their maximum range $\left(\Delta_{\max } T\right)$ (dashed curves). During an orbital period of $\sim 100$ minutes, there are intervals of thermal warming and cooling corresponding to sunlit and dark portions of the orbit. A regular orbital oscillation is usually superimposed upon irregular short-term perturbation caused by solar contamination marked by the vertical dotted lines.

The middle panels $(b-c)$ show the variation in gain for channels 3 and 5, derived according to NOAA calibration procedures (solid lines). During the periods of solar contamination, the gains experience considerable perturbations. For channel 3, NOAA applies a special procedure to flag the solar contamination events and replaces the corrupted gains and offsets by constant values. This is seen as the plateaus in $\mathbf{G}^{(3)}$ curves. No such correction is currently employed for IR channels 4 and 5. Dashed lines on panels $(c)$ depict channel 5 gain $\mathbf{G}^{(5)}$, where irregularities were filtered out according to the method proposed and discussed later.

The bottom panels $(d)$ show the difference $\left(C_{I C T}-C_{S P}\right)$ for channels 3 and 5 .
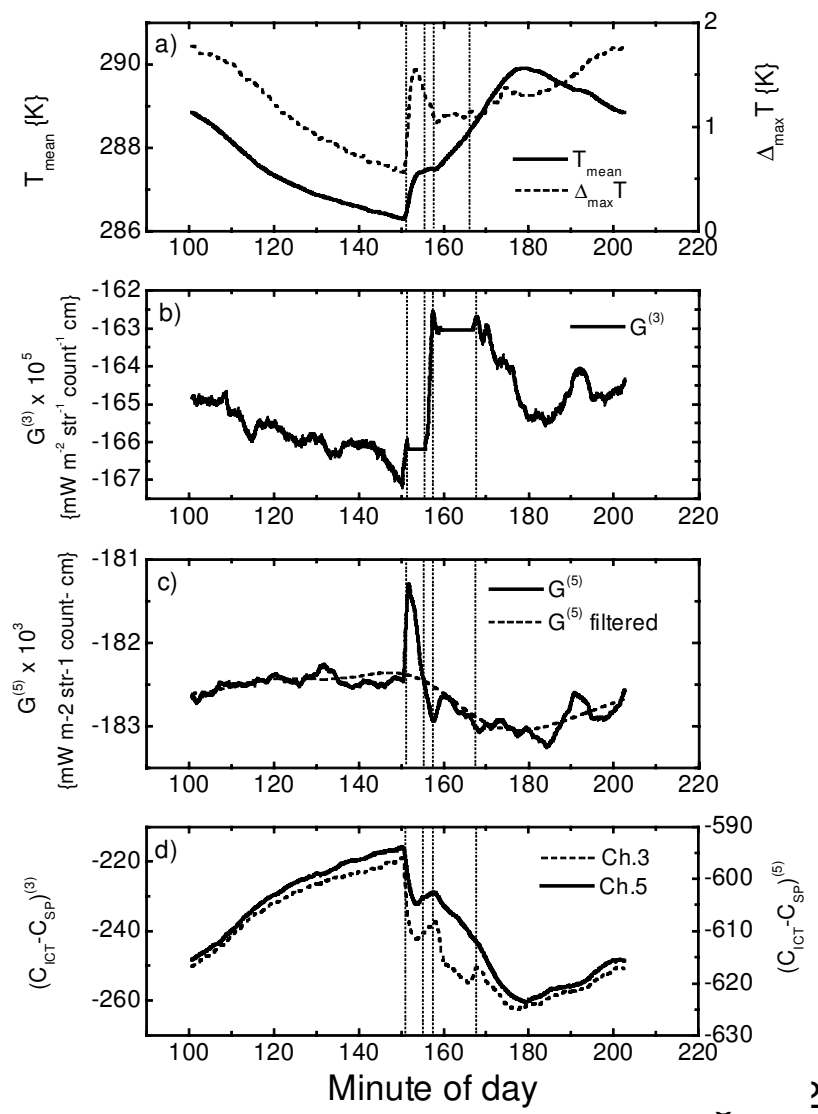

Figure 2. Same as figure 1 but for AVHRR/NOAA-14 on 22 December 1997. 
A high correlation is observed between the two channels, indicating significance of solar contamination for thermal channel 5 . The same effect is observed for channel 4 (not shown). Although these channels do not register any solar radiation directly, their readings are affected by thermal heating due to absorption of solar radiation by the ICT surface.

\section{Method for correcting IR calibration}

The perturbation in the gain is a manifestation of inadequate simulation of radiation from the ICT (Steyn-Ross et al. 1992). Two possible causes are (1) unaccounted solar radiation impinging in the AVHRR interior and (2) inability to adequately track rapid changes in the ICT temperature due to the PRT thermal inertia. While both effects are important for channel 3, only the second one is essential for channels 4 and 5 .

To correct for solar contamination of the calibration data for channels 4 and 5 , we propose to filter out high-frequency harmonics in the time series of the gain. This is done for 1 orbital cycle of calibration data, when solar contamination is detected, by conducting a forward Fourier transform, dropping harmonics with periods shorter than a pre-determined threshold and then applying a backward Fourier transform to reconstruct a smoother temporal trend. A similar method is recommended for
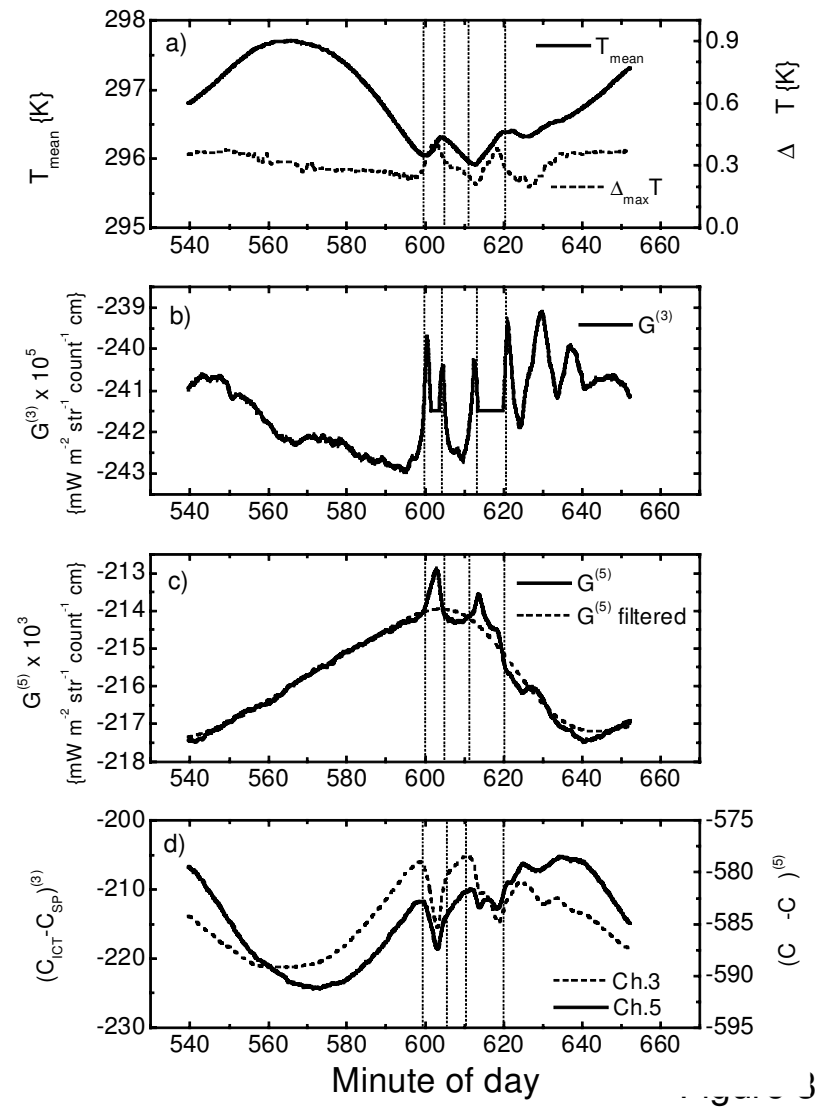

Figure 3. Same as figures 1 and 2, but for AVHRR/NOAA-15 on 1 September 1999. Gains are recomputed from three operational calibration parameters $a_{0,1,2}$ given in GAC data. 
channel 3 to improve the current operational correction, which is based on constant threshold. The middle panels $(c)$ of figures $1-3$ show the smoothed gain curves obtained by filtering out high-frequency components of period shorter than 15 minutes. This value was selected as a separation between signal and noise following analyses of the power spectra. The physical reasoning for removing the highfrequency harmonics lies in the large thermal inertia of the AVHRR subsystems, which have response time ranging from 30 minutes to several hours (Walton et al. 1998). Moreover, the entire AVHRR detector system is maintained at a constant temperature around $107 \mathrm{deg} \mathrm{K}$ (Cracknell 1997). All these were designed to prevent sudden changes in the gains, like those shown in figures 1-3.

The filtering procedure may be applied iteratively, as shown in figure 1 for AVHRR/NOAA-12. This can suppress more effectively the influence of the irregular behaviour, which contributes to the amplitudes of all harmonics. The iterative process converges quickly.

\section{Discussion}

Solar contamination has a significant impact on SST mapping. Figure 4 (left panels) shows a latitudinal variation of the temperature correction $\Delta T=T_{\text {cor }}-T_{\text {mean }}$,
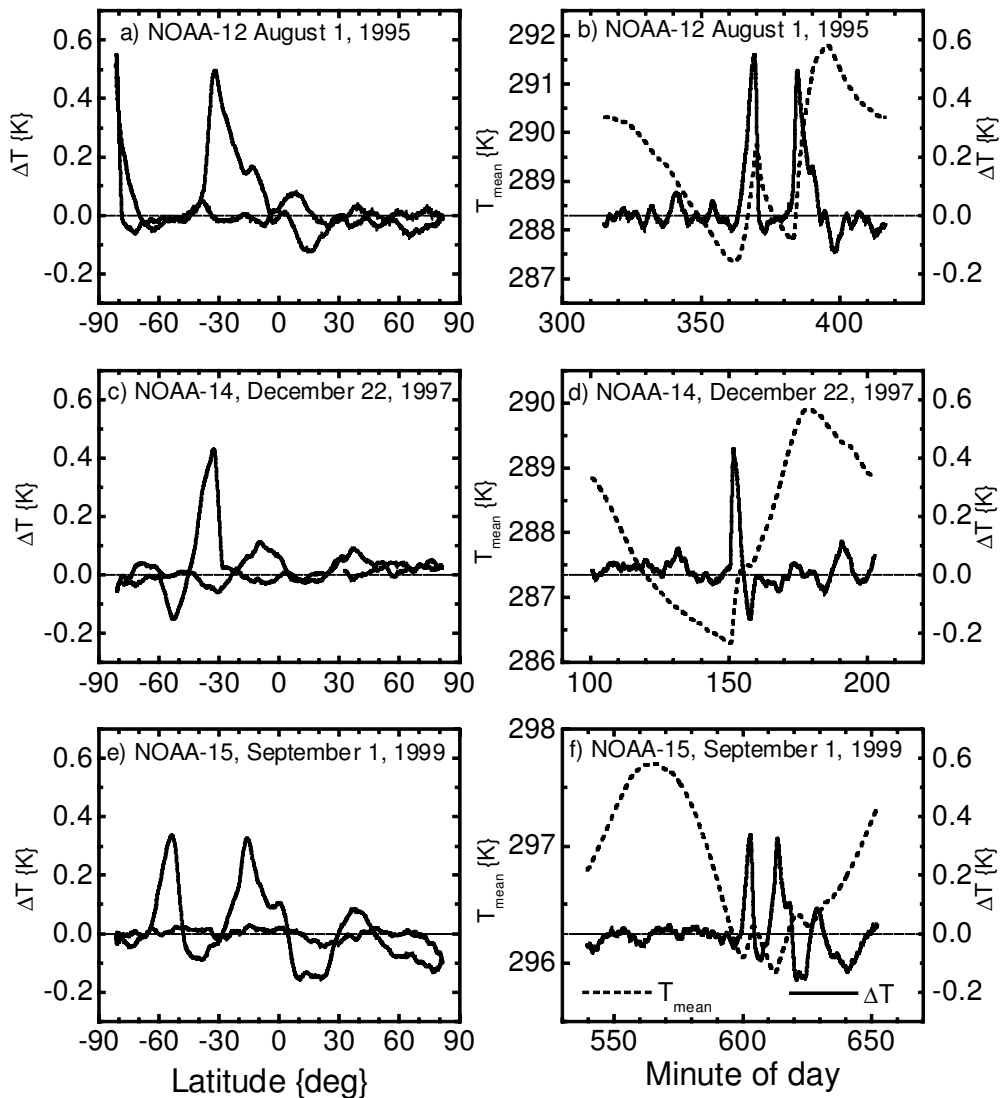

Figure 4. Left: Latitudinal dependence of the temperature correction due to solar contamination of the calibration of channels 4 and 5. Right: Temporal variations of the mean ICT temperature $T_{\text {mean }}$ measured by the PRTs (dashed) and temperature correction $\Delta T$ (solid). 
where $T_{c o r}$ is a corrected $\mathbf{T}_{I C T}$ using modified gains. We estimated $T_{\text {cor }}$ by averaging the temperature corrections for channels 4 and 5 . They are usually within $\pm 0.1 \mathrm{deg} \mathrm{K}$ of each other. The largest effect (up to $0.6 \mathrm{deg} \mathrm{K}$ ) is observed for the AVHRR/ NOAA-12. Due to the nature of observation by polar orbiting satellites, the effect causes biases in the brightness temperature over large areas of the globe. SST derived in these zones is systematically lower and may be confused with negative climatic signal. There are also areas of opposite effect, though its amplitude is usually less than $0.2 \mathrm{deg} \mathrm{K}$. In the dark part of the orbit, the temperature correction is usually negligible (typically less than $0.1 \mathrm{deg} \mathrm{K}$ ).

The right panels of figure 4 show the linkage between $T_{\text {mean }}$ and $\Delta T$. There is a time lag between curves during the perturbation, with $\Delta T$ advancing $T_{\text {mean }}$ by several minutes. This ratifies our explanation of the origin of the artificial gain variation as a result of thermal inertia in the PRT data.

\section{Acknowledgments}

The authors thank Gunar Fedosejevs for his valuable comments and discussion.

\section{References}

Allen, M. R., Mutlow, C. T., Blumberg, G. M. C., Christy, J. R., McNider, R. T., and Llewellyn-Jones, D. T., 1994, Global change detection. Nature, 370, 24-25.

Brown, O. B., Brown, J. W., and Evans, R. H., 1985, Calibration of Advanced Very High Resolution Radiometer infrared observations. Journal of Geophysical Research, 90, $11667-11677$.

Brown, J. W., Brown, O. B., and Evans, R. H., 1993, Calibration of Advanced Very High Resolution Radiometer infrared channels: A new approach to nonlinear correction. Journal of Geophysical Research, 98, 18 257-18 268.

Cracknell, A. P., 1997, The Advanced Very High Resolution Radiometer (AVHRR) (London: Taylor \& Francis).

KIDwell, K. B. (editor), 1998, NOAA Polar Orbiter Data User's Guide. US Department of Commerce, NESDIS, NOAA, National Climatic Data Center, Satellite Data Services Division, Washington, DC, USA.

Steyn-Ross, D. A., Steyn-Ross, M L., and Clift, S., 1992, Radiance calibration of Advanced Very High Resolution Radiometer infrared channels. Journal of Geophysical Research, 97, 5551-5568.

Walton, C. C., Sullivan, J. T., Rao, C. R. N., and Weinreb, M. P., 1998, Corrections for detector nonlinearities and calibration inconsistencies of the infrared channels of the Advanced Very High Resolution Radiometer. Journal of Geophysical Research, 103, $3323-3337$. 